\title{
Performance analysis of OFDM-IM scheme under STO and CFO
}

\author{
Suyoto $^{1}$, Agus Subekti ${ }^{2}$, Arief Suryadi Satyawan ${ }^{3}$, Vita Awalia Mardiana ${ }^{4}$, Nasrullah Armi, \\ Dayat Kurniawan $^{6}$ \\ 1,2,3,4,5,6 Research Center for Electronics and Telecommunication, Lembaga Ilmu Pengetahuan Indonesia (LIPI), Indonesia \\ ${ }^{3}$ Electrical Engineering Department, Nurtanio University, Indonesia
}

\section{Article Info}

Article history:

Received Aug 26, 2020

Revised Dec 11, 2020

Accepted Jan 13, 2021

\section{Keywords:}

$\mathrm{CFO}$

Frequency-selective channel

ICI

OFDM-IM

STO

\begin{abstract}
In this letter, performance analysis of orthogonal frequency division multiplexing with index modulation (OFDM-IM) is presented in term of bit error rate (BERs). The analysis considers its performance under two impairments, symbol time offset (STO) and carrier frequency offset (CFO) in frequency-selective fading channel. As orthogonal multicarrier system, OFDM-IM is subject to both inter-symbol interference (ISI) and inter-carrier interference (ICI) in a frequency-selective fading channel. OFDM-IM is a new multicarrier communication system, where the active subcarriers indices are used to carry additional bits of information. In general, in the previous existing works, OFDM-IM are evaluated only for near-ideal communication scenarios by only incorporating the CFO factor. In this work, the OFDM-IM performance is investigated and compared with conventional OFDM in the presence of two impairments, STO and CFO. Simulation results show that OFDM-IM outperforms the conventional OFDM with the presence of STO and CFO, especially at high SNR areas.
\end{abstract}

This is an open access article under the CC BY-SA license.

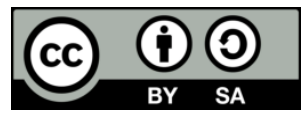

\section{Corresponding Author:}

Suyoto

Research Center for Electronics and Telecommunication

Lembaga Ilmu Pengetahuan Indonesia (LIPI)

LIPI Complex, Building 20. Floor $4^{\text {th }}$ Cisitu street no. 21/154D Bandung 40135

Email: suyo003@lipi.go.id

\section{INTRODUCTION}

Orthogonal frequency division multiplexing (OFDM) is a multicarrier transmission that excels in handling inter-symbol interference (ISI) caused by multipath fading. This allows high speed transmission so that this technique has been adopted in many wireless communication standards [1-8]. The next OFDM development is OFDM with index modulation (OFDM-IM). OFDM-IM is a new OFDM scheme where information transmission is carried out by the $\mathrm{M}$-ary signal constellation and also using a subcarrier index. The subcarrier index is activated according to the relevant information bits [9-11]. By adjusting the number of active subcarriers in the OFDM-IM scheme, this technique provides an interesting trade-off between performance and spectral efficiency [9].

The use of index modulation techniques has also been expanded to direct-sequence spread spectrum (DSSS) modulation techniques to thrift spectrums efficiency and to achieve higher data rates with lower complexity $[12,13]$. Even so, as a multicarrier systems, which is alike to conventional OFDM; where performance is affected by a symbol time offset (STO) $[2,14,15]$ and carrier frequency offset (CFO) $[2,16-21]$. In particular, OFDM-IM is even very susceptible to CFO as indicated by [22-24]. 
Wide-ranging of analysis and simulation studies were discussed in $[2,14,15,20,21]$ to describing the effects of STO and CFO in conventional OFDM systems. In [20], studied the impact of STO and CFO on OFDM performance under the AWGN channel. In [21], the authors reproduce the approximation method [20] into a frequency-selective fading channel for some errors due to CFO that improves work assessment (BERs). In $[2,14,15]$, STO causes ISI and inter-carrier interference (ICI) for the frequency-selective fading channel. Moreover, ICI can significantly degrade the channel estimation performance [25-29].

Since OFDM-IM adopts block-based modulation, while existing contributions assume freedom from the OFDM subcarriers, it may not be directly applicable to the OFDM-IM scheme. The authors [22] examined the OFDM-IM signal-to-interference ratio (SIR) under Doppler spread that caused CFO to deploy scenarios under a simplified AWGN channel rather than a more practical frequency-selective fading channel. The authors [23] derived analysis and simulation of the OFDM-IM scheme's performance under ICI conditions in the frequency-selective fading channel. The authors [24] analyzed and compared the OFDM-IM scheme's performance in conditions under ICI and in-phase/quadrature (IQ) imbalance.

In this letter, we simulate the OFDM-IM scheme's performance under STO and CFO, where STO can also cause ISI and ICI in a frequency-selective fading channel. Apart from STO, ICI can also be caused by CFO. Even though the current OFDM-IM scheme is evaluated and compared from the perspective of spectrums efficiency, CFO, and IQ; error performances of the OFDM-IM scheme under STO is yet unknown. Hence, this letter aims to analyze and compare the OFDM-IM scheme with conventional OFDM under the influence of STO and CFO in a frequency-selective Rayleigh fading channel.

The rest of this letter is arranged as follows: Section 2 provides a signal model for OFDM-IM scheme. Furthermore, mathematical models of STO and CFO are concisely discussed. Section 3 provides related work and section 4 provides simulation results and discussions. Finally, section 5 concludes the letter.

\section{OFDM-IM SIGNAL MODEL}

In this section, the OFDM-IM scheme is described first. After that, a mathematical model of the signal received under STO and CFO will be given.

\subsection{OFDM-IM scheme}

In the conventional OFDM scheme, several $N$ subcarriers are used to transmit information from transmitter to receiver, which operates in the frequency-selective Rayleigh fading channel. The number of bits of information carried is $t=N \times \log _{2}(M)$, where $M$ denotes the modulation order. $X$ frequency domain signals are obtained after the information bits are mapped to the subcarrier through a serial to parallel converter process. After that, inverse fast fourier transform (IFFT) is used on $X$ to get OFDM signal in the time domain as (1).

$$
x(n)=\sum_{k=0}^{N-1} X(k) e^{\frac{j 2 \pi n k}{N}}, 0 \leq n \leq N-1 .
$$

The transmitted signal is sent via the frequency-selective Rayleigh fading channel as (2).

$$
y(n)=\sum_{l=0}^{L-1} x(n-l) h(l)+w(n),
$$

with $h(l)$ represents the channel response, $L$ denotes the number of channel paths, and $w(n)$ represents complex Gaussian noise with zero mean and variance $\sigma_{0}^{2}$. At the receiver, the relationship between inputoutput in the frequency domain for the $k$-th subcarrier can be described as (3).

$$
Y(k)=X(k) H(k)+W(k)
$$

with $H(k)$ and $W(k)$ denote the frequency response of channel $h(n)$ and noise $w(n)$, respectively. To prevent ISI in the OFDM scheme, a cycle prefix (CP) with length of $C_{p}$ is added on $x(n)$.

In contrast to the conventional OFDM, OFDM-IM carries data information on the modulated subcarrier and the active subcarrier indices. In OFDM-IM, the OFDM block of size $N$ is divided into $G$ subblocks with $m=N / G$ subcarriers per subblock. The number of subcarriers is assigned some $q$ from $m$ per subblock. The active subcarriers' indices carry $p 1=\left\lfloor\log _{2} C(m, q)\right\rfloor$ bits, where $C(m, q)$ and $\lfloor$.$\rfloor denote$ the binomial coefficient and floor function, respectively. Some $p 2=q \times \log _{2}(M)$ bits are converted to $M$ ary symbols $s_{i}$ and are carried by active subcarriers, where $s_{i} \in\left[s_{1}, s_{2}, \ldots, s_{M}\right]$. Therefore, the total number of data bits sent in one OFDM-IM block is as (4). 


$$
t=G p=G(p 1+p 2)
$$

where $p=p 1+p 2$ is the number of bits per OFDM-IM subblock. Figure 1 shows the block diagram of OFDM-IM transmitter.

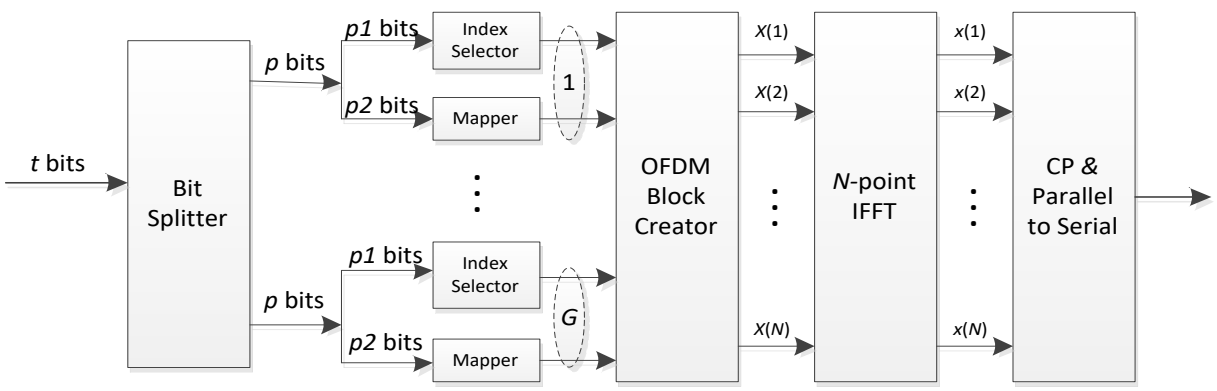

Figure 1. Block diagram of the OFDM-IM transmitter [9]

\subsection{STO model}

In the OFDM-IM, just like the conventional OFDM, IFFT and FFT functions are needed for modulation and demodulation in each transmitter and receiver, respectively. To obtain one block of OFDMIM symbol with several $N$ samples in the receiver, it is necessary to have a proper samples of the transmitted signal for the duration of the OFDM-IM symbol. Therefore, time synchronization is required to obtain a proper samples of each OFDM-IM symbol, where in this process has also discarded the CP. Hence, the signal received in the time domain under STO can be written (5).

$$
\hat{y}(n)=y(n-\delta)
$$

where $\delta$ denotes the STO. The frequency response of $\hat{y}(n)$ equals [2].

$$
\begin{aligned}
& \hat{Y}(k)=\frac{N-\delta}{N} X(i) e^{\frac{j 2 \pi i \delta}{N}}+\sum_{i=0, i \neq k}^{N-1} X(i) e^{\frac{j 2 \pi i \delta}{N}} \sum_{n=0}^{N-1-\delta} e^{\frac{j 2 \pi(i-k) n}{N}}+ \\
& \frac{1}{N} \sum_{i=0}^{N-1} X_{l}(i) e^{\frac{j 2 \pi i\left(2 \delta-C_{p}\right) / N}{N}} \sum_{n=N-\delta}^{N-1} e^{j 2 \pi(i-k) n / N},
\end{aligned}
$$

where:

$$
\sum_{n=0}^{N-1-\delta} e^{\frac{j 2 \pi(i-k) n}{N}}=e^{j \pi(i-k) \frac{N-1-\delta}{N}} \cdot \frac{\sin \left(\frac{(N-\delta) \pi(k-i)}{N}\right)}{\sin \left(\frac{\pi(k-i)}{N}\right)}=\left\{\begin{array}{cl}
N-\delta & \text { for } i=k \\
\text { Nonzero } & \text { for } i \neq k
\end{array}\right.
$$

with $i, k \in\{0,1, \ldots, N-1\}$. The second term in (6) correlate with ICI, which states that the orthogonality has been ruined. In the third term in (6), it is also clear that the received signal exist ISI (of the OFDM symbol in front of it $\left(X_{l}\right)$ ).

\subsection{CFO model}

CFO is generally caused by two types of interference [17], the first is the phase noise caused by instability of the carrier signal generators used at the transmitter and receiver. This phase noise can be modeled by a zero-mean Wiener random process as shown in $[18,19]$. The second is CFO, which is caused by the Doppler frequency. Hence, the signal received in the time domain under CFO can be written as (7).

$$
\hat{y}(n)=y(n) e^{\frac{j 2 \pi n \varepsilon}{N}}
$$

where $\varepsilon$ denotes normalized CFO (NCFO) to subcarrier spacing. The frequency response of $\hat{y}(n)$ equals [2].

$$
\hat{Y}(k)=\frac{\sin (\pi \varepsilon)}{N \sin \left(\frac{\pi \varepsilon}{N}\right)} e^{\frac{j \pi \varepsilon(N-1)}{N}} H(k) X(k)+I(k)+W(k),
$$


where:

$$
I(k)=e^{\frac{j \pi \varepsilon(N-1)}{N}} \sum_{i=0, i \neq k}^{N-1} \frac{\sin (\pi(i-k)+\varepsilon))}{N \sin (\pi(i-k+\varepsilon) / N)} H(i) X(i) e^{j \pi(i-k)(N-1) / N},
$$

The first term in (8) denotes the amplitude and phase distortion of the $k$-th subcarrier frequency component due to CFO. The second term $(I(k))$ in (8) denotes the ICI from other subcarriers into the $k$-th subcarrier frequency component. In this situation, the orthogonality among subcarrier frequency components is not preserve any more due to the CFO.

\section{RELATED WORK}

The authors in [22] examined the OFDM-IM signal-to-interference ratio (SIR) under Doppler spread that caused CFO to deploy scenarios under AWGN channel. It was found that the theoretical values and simulation results are almost the same, and the SIR performance for OFDM-IM modulation is around $3 \mathrm{~dB}$ better than OFDM modulation. In [23], the authors derived analysis and simulation of the OFDM-IM scheme's performance in conditions under ICI in the frequency-selective fading channel. The authors in [23] proposed a different approach on the influence of ICI on OFDM-IM based on subblocks (the intra-subblock and the inter-subblocks) and found that OFDM-IM outperformed conventional OFDM with CFO. In [24], the authors analyzed and compared the OFDM-IM scheme's performance with other multicarrier scheme in conditions under ICI and IQ imbalance. They found that OFDM with generalized IM (OFDM-GIM) offers the highest spectrums efficiency, being the most susceptible to IQ effects. OFDM with subcarrier number modulation (OFDM-SNM) offers worse BERs performance than OFDM-IM under the imbalance of CFO and IQ due to sequential subcarrier activation.

\section{SIMULATION RESULTS AND DISCUSSION}

The performance of the OFDM-IM and conventional OFDM systems was investigated under the influence of STO and CFO. The simulation parameters are given in Table 1. For OFDM-IM, the number subcarriers per subblock $(m)$ is set to 4 , so that $G=N / 4$, active subcarriers used per subblock $(q)$ is set to 2 , and maximum likelihood detector [9] is used for active subcarriers detection. The parameter of STO = 0 indicates the correct arrival time of the OFDM/OFDM-IM symbol.

Table 1. The simulation parameters

\begin{tabular}{lll}
\hline No. & Parameter & Values \\
\hline 1. & Channel model & Vehicular B [30] \\
2. & Number of FFT $(N)$ & 2048 \\
3. & $C_{p}$ & 220 \\
4. & Data modulation & 4 -quadrature amplitude modulation (4-QAM) \\
5. & Number of bit per Symbol $(t)$ & 3072 \\
6. & Sampling rate & $0.1 \mu \mathrm{s}$ \\
7. & STO $(\delta)$ & $0,-100,10$ \\
8. & NCFO $(\varepsilon)$ & $0.04,0.08,0.12$ \\
9. & Number of symbol per simulation & 5000 \\
\hline
\end{tabular}

We set the STO distance for the STO parameters before the correct arrival time to be much greater than the STO after the correct arrival time. This is done to ensure the STO before the OFDM/OFDM-IM symbol's correct arrival time contains ISI from the previous OFDM symbol; since the STO before the correct arrival time of the OFDM/OFDM-IM symbol is in the CP areas. We use the same training symbol for channel estimation for multicarrier systems generated from real pseudo-noise sequence placed in even subcarriers and give zero values for odd subcarriers. The Least Squared algorithm [2] is used for the channel estimation.

In Figure 2 shows the results of the conventional OFDM and the OFDM-IM simulations with the value of $N C F O=0.04$. The OFDM-IM has better performance than the conventional OFDM at $S T O=10$, while at STO $=0$ and $S T O=-100$, the conventional OFDM performance is slightly better than the OFDMIM. This shows that the OFDM-IM is more resistant to STO interference, especially the STOs greater than 0 . The STOs in this area are more significant, causing ISI and ICI than STOs smaller than 0 (protected by CP).

In Figure 3, the results of the conventional OFDM and the OFDM-IM simulations with the value of $N C F O=0.08$. OFDM-IM has better performance than conventional OFDM on all tested STOs, namely $S T O=10, S T O=-100$, and $S T O=0$, especially at high signal-to-noise ratio (SNR) $(\mathrm{SNR} \geq 20 \mathrm{~dB}$ ). 
Figure 4 shows the results of the conventional OFDM and OFDM-IM simulations with the value of $N C F O=0.12$. OFDM-IM also performs better than conventional OFDM on all tested STOs, namely $S T O=10, S T O=-100$, and $S T O=0$, especially at high SNR (SNR $\geq 15 \mathrm{~dB})$. This shows that OFDM-IM is more resistant against ICI interference caused by CFO, where the more significant the CFO, the performance of OFDM-IM is better than conventional OFDM. This is because the number of subcarriers used (modulated subcarriers) on OFDM-IM is smaller than conventional OFDM with the same number of bits transmitted. Note that the BERs results are obtained without STO and CFO compensation in the receiver.

From the test results on the Vehicular B [30] channel, where this channel is commonly used for modeling mobile communications, it can be concluded that STO can cause ISI and ICI, especially at STO $>0$. Likewise, with the CFO, which worsens the ICI. The presence of ISI and ICI can reduce the performance in both conventional OFDM and OFDM-IM systems. From the performance evaluation results, it was found that OFDM-IM had better performance than conventional OFDM with the presence of STO and CFO, especially at high SNR areas (SNR $\geq 15 \mathrm{~dB}$ ). However, at low SNR, the detection of active subcarriers on OFDM-IM experiences an error so that the bit error rate increases at low SNR. Therefore, more accurate detection of active subcarriers is needed to improve OFDM-IM performance, especially at low SNR. This study does not yet involve IQ imbalance effects, so future studies may involve the effects of IQ imbalance together with the effects of STO and CFO on the OFDM-IM scheme.

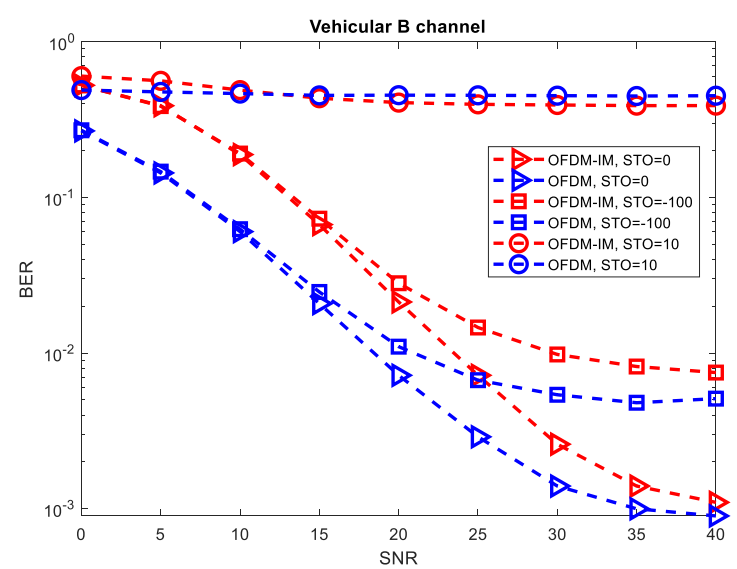

Figure 2. Comparison of BERs of OFDM-IM and conventional OFDM with $\mathrm{NCFO}=0.04$

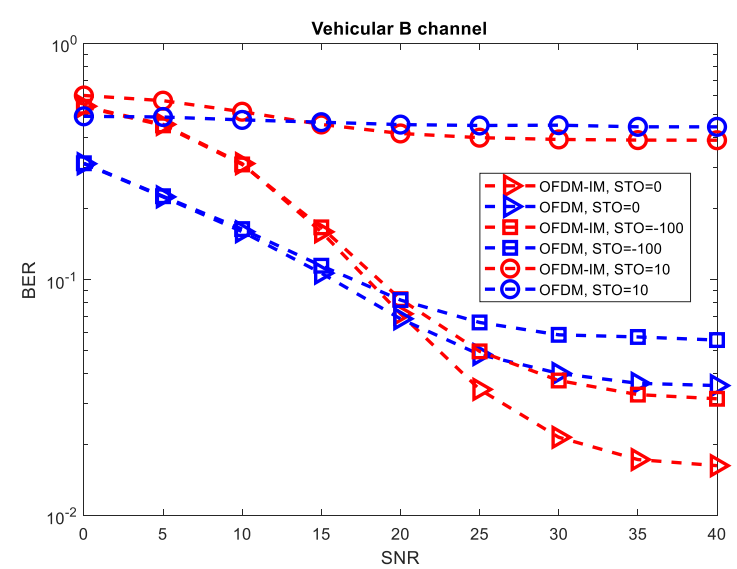

Figure 3. Comparison of BERs of OFDM-IM and conventional OFDM with $\mathrm{NCFO}=0.08$

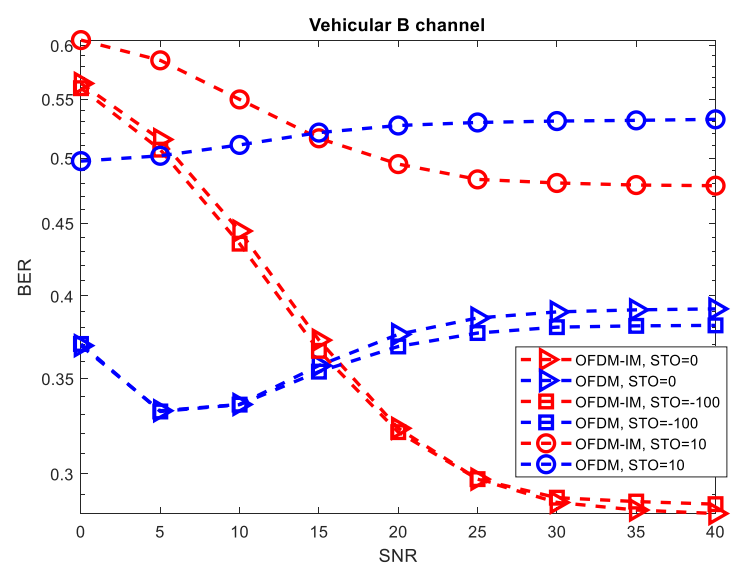

Figure 4. Comparison of BERs of OFDM-IM and conventional OFDM with NCFO=0.12

\section{CONCLUSION}

In this letter, we have provided an analysis and computer simulations of the OFDM-IM disturbances caused by STO and CFO compared with the conventional OFDM, where STO causes ISI and ICI, and CFO also causes ICI. The simulation results show that the OFDM-IM performance is better than the conventional 
OFDM in high STO and CFO conditions and in the high frequency-selective Rayleigh fading channel, especially in high SNR areas (SNR $\geq 15 \mathrm{~dB}$ ). Therefore, OFDM-IM is suitable for high data rates communications and high mobility and spectrum allocation, which is more flexible than conventional OFDM.

\section{ACKNOWLEDGEMENTS}

The authors thank the editors and anonymous reviewers for their comments and suggestions which have been very helpful in improving the grade of this letter, and we also thank to the Indonesia Endowment Fund for Education (LPDP) for their endorsement to our works in this research.

\section{REFERENCES}

[1] B. Sklar, "Digital Communications: Fundamentals and Applications 2/E," Prentice Hall, 2002.

[2] Y. S.Cho, J. Kim, W. Y. Yang, and C. G. Kang, "Mimo-OFDM Wireless Communications with Matlab," Wiley and Sons (Asia) Pte Ltd., 2010.

[3] H. Bogucka, A. Kliks, and P. Kryszkiewicz, "Advanced Multicarrier Technologies for Future Radio Communication," John Wiley \& Sons, Inc., 2017.

[4] T. Hwang and C. Yang, "OFDM and Its Wireless Applications: A Survey," IEEE Transaction on Vehicular Technology, vol. 58, no. 4, pp. 1673-1694, 2009.

[5] Shivapanchakshari T. G. and H. S. Aravinda, "CL-SA-OFDM: cross-layer and smart antenna based OFDM system performance enhancement," International Journal of Electrical and Computer Engineering (IJECE), vol. 10, no. 5, pp. 4663-4670, 2020.

[6] "IEEE Standard for Information Technology-Telecommunications and information exchange between systemsLocal and Metropolitan networks-Specific requirements-Part 11: Wireless LAN Medium Access Control (MAC) and Physical Layer (PHY) specifications: Higher Speed Physical Layer (PHY) Extension in the $2.4 \mathrm{GHz}$ band," in IEEE Std 802.11b-1999, 1999.

[7] "ETSI, Digital video broadcasting (DVB): Frame structure, channel coding and modulation for a second generation digital terrestrial television broadcasting system (DVB-T2)," Tech. Rep. ETSI EN 302755 V1.1.1, 2009.

[8] IEEE 802.16m-09/0034r4 IEEE 802.16m System Description Document [Draft], 2010.

[9] E. Basar, U. Aygolu, E. Panayirci, and H. V. Poor, "Orthogonal frequency division multiplexing with index modulation," IEEE Transactions on Signal Processing, vol. 61, no. 22, pp. 5536-5549, 2013.

[10] F. Rui, Y. J. Yu, and Y. L. Guan, "Generalization of orthogonal frequency division multiplexing with index modulation," IEEE Transactions on Wireless Communications, vol. 14, no. 10, pp. 5350-5359, 2015.

[11] M. Wen, X. Cheng, and M. Ma, "On the achievable rate of OFDM with index modulation," IEEE Transactions on Signal Processing, vol. 64, no. 8, pp. 1919-1932, 2015.

[12] G. Kaddoum, M. F. A. Ahmed, and Y. Nijsure, "Code index modulation: A high data rate and energy ffficient communication system," IEEE Communications Letters, vol. 19, no. 2, pp. 175-178, 2015.

[13] G. Kaddoum, Y. Nijsure, and H. Tran, "Generalized code index modulation technique for high data rate communication systems," IEEE Transactions on Vehicular Technology, vol. 65, no. 9, pp. 7000-7009, 2016.

[14] Y. Mostofi and D. C. Cox, "Mathematical Analysis of the Impact of Timing Synchronization Error on the Performance of an OFDM System," IEEE Transactions on Communications, vol. 54, no. 2, pp. 226-230, 2006.

[15] A. Al-Dweik, S. Younis, A. Hazmi, C. Tsimenidis and B. Sharif, "Efficient OFDM Symbol Timing Estimator Using Power Difference Measurements," IEEE Transactions on Vehicular Technology, vol. 61, no. 2, pp. 509-520, 2012.

[16] A. Al-Dweik, R. Hamila and M. Renfors, "Blind Estimation of Large Carrier Frequency Offset in Wireless OFDM Systems," IEEE Transactions on Vehicular Technology, vol. 56, no. 2, pp. 965-968, 2007.

[17] C. Muschallik, "Influence of RF oscillator on an OFDM signal," IEEE Transactions on Consumer Electronics, vol. 41, no. 3, pp. 592-603, 1995

[18] T. Pollet and M. Moeneclaey, "Synchronizability of OFDM signals," Proceedings of GLOBECOM '95, Singapore, 1995, pp. 2054-2058, vol. 3.

[19] L. Tomba, "On the effect of Wiener phase noise in OFDM systems," IEEE Transactions on Communications, vol. 46, no. 5, pp. 580-583, 1998.

[20] T. Keller and L. Hanzo, "Adaptive multicarrer modulation: A convenient framework for time-frequency processing in wireless communications," Proceedings of the IEEE, vol. 88, no. 5, pp. 611-640, 2000.

[21] L. Rugini and P. Banelli, "BER of OFDM systems impaired by carrier frequency offset in multipath fading channels," IEEE Transactions on Wireless Communications, vol. 4, no. 5, pp. 2279-2288, 2005.

[22] Y. S. Yu, Y. Feng, K. M. Kim, and S. C. Kim, "Performance analysis of subcarrier index modulation OFDM in Doppler spread environments," 2016 7th International Conference on Intelligent Systems, Modelling and Simulation (ISMS), Bangkok, 2016, pp. 380-382.

[23] Q. Ma, P. Yang, Y. Xiao, H. Bai, and S. Li, "Error probability analysis of OFDM-IM with carrier frequency offset," IEEE Communications Letters, vol. 20, no. 12, pp. 2434-2437, 2016.

[24] A. Tusha, S. Dogan, and H. Arslan, "Performance analysis of frequency domain IM schemes under CFO and IQ imbalance," 2019 IEEE 30th Annual International Symposium on Personal, Indoor and Mobile Radio Communications (PIMRC), Istanbul, Turkey, 2019, pp. 1-5.

[25] T. S. Rappaport, "Wireless Communications: Principles and Practice 2/E," Prentice Hall, 2001. 
[26] D. Greenwood and L. Hanzo, "Characterization of mobile radio channels," Mobile Radio Communications (ed. R. Steele), Pentech Press-IEEE Press, London, 1994.

[27] Maryam K. Abboud, Bayan M. Sabbar, "Performance evaluation of high mobility OFDM channel estimation techniques," International Journal of Electrical and Computer Engineering (IJECE), vol. 10 no. 3, pp. 2562-2568, 2020.

[28] H. Hamlili, S. Kameche, A. Abdelmalek, "S $\alpha$ S noise suppression for OFDM wireless communication in rayleight channel," International Journal of Electrical and Computer Engineering (IJECE), vol. 10 no. 2, pp. 2003-2010, 2020.

[29] N. Shaik, P. K. Malik, "A Retrospection of Channel Estimation Techniques for 5G Wireless Communications: Opportunities and Challenges," International Journal of Advanced Science and Technology, vol. 29 no. 5, pp. 8469-8479, 2020.

[30] “Guidelines for evaluation of radio transmission technologies for IMT-2000,” Recommendation ITU-R M. 1225, 1997.

\section{BIOGRAPHIES OF AUTHORS}
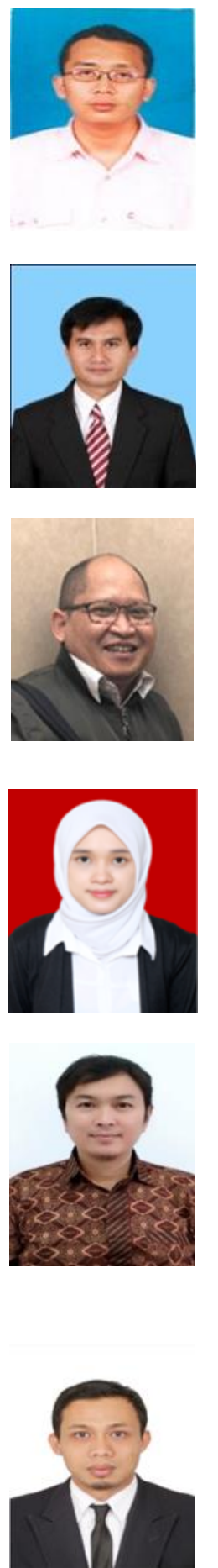

Suyoto is currently a researcher at Research Center Electronics and Telecommunication Indonesian Institute of Sciences (LIPI). Obtained Bachelor of Telecommunication Engineering, Master in Computer and Multimedia Systems, and Doctor of Telecommunication Engineering all from the Bandung Institute of Technology in 2002, 2009 and 2019, respectively. His research interests include signal processing, synchronization, multicarrier system, and wireless communication.

Agus Subekti got bachelor and master degree in electrical engineering from Bandung Institute of Technology, Indonesia, in 1998 and 2001 respectively. He finished his Ph.D. in electrical engineering \& informatics from Bandung Institute of Technology (ITB), in 2016. He was a research associate at Nakajima Laboratory, Tokai University, Japan in 2002-2004. Currently, he is a researcher with Indonesian Institute of Sciences since 2005.

Arief Suryadi Satyawan has joined with Research Center for Electronics and Telecommunication, Indonesian Institute of Science, since 1996. He received his Bachelor's degree in electrical engineering from Universitas Jenderal Achmad Yani Bandung, Indonesia, in 2000. In 2007, he received his Master's degree in electrical engineering from Institut Teknologi Bandung, Indonesia. He got his Ph.D. in computer and communication engineering from Waseda University, Japan, in 2019. His current research is image and video processing, and video communication system. He is a member of The Institute of Image Information and Television Engineers (ITE), Japan, and Information Processing Society of Japan (IPSJ).

Vita Awalia Mardiana received M.Sc. degree on Microelectronics engineering, in School of Electrical Engineering and Informatics Institut Teknologi Bandung, Indonesia in 2018. Currently she is working as a researcher at Research Center Electronic and Telecommunication Indonesian Institute of Sciences (LIPI). Her research interests include digital signal processing, embedded system, wireless communication, and machine learning.

Nasrullah Armi received Master Degree from Department of Knowledge based Information Engineering, Toyohashi University of Technology, Japan in 2004. Subsequently, he obtained his Ph.D degree from Department of Electrical and Electronic Engineering, Universiti Teknologi Petronas, Malaysia in 2013. His research interests are Signal Processing, Wireless Communication and Networks. He published scientific articles on peer review journals and participated on international conferences. He contributed his knowledge as scientific reviewer in international impact journals and became Scientific Committee in International Conferences. Currently, he is working as a researcher at Indonesian Institute of Sciences, Research Center for Electronics \& Telecommunication

Dayat Kurniawan received M.Sc. degree on Telecommunication engineering, in School of Electrical Engineering and Informatics Institut Teknologi Bandung, Indonesia in 2019. Currently he is working as a researcher at Research Center Electronics and Telecommunication Indonesian Institute of Sciences (LIPI). His research interests include signal processing, wireless communication, and embedded system. 